\title{
COMBINING ABILITY ANALYSIS FOR ACHENE YIELD AND RELATED TRAITS IN SUNFLOWER (Helianthus annuus L.)
}

\author{
Muhammad Waqas Ahmad ${ }^{1}$, Muhammad Shahzad Ahmed ${ }^{1 *}$, and Hammad Nadeem Tahir ${ }^{1}$
}

Ten sunflower (Helianthus annuus L.) lines, five testers, and 50 crosses developed in line $\times$ tester fashion were evaluated for general combining ability (GCA) and specific combining ability (SCA) effects in a triplicate randomized complete block design, in Faisalabad, during 2009-2010. Genetic variability among genotypes was assessed for days to flowering, days to maturity, plant height, internodal length, leaf area, number of leaves per plant, head diameter, stem girth, percentage of filled achenes, 100 achene weight, achene yield per plant. A-1, A-7, A-27 and A-39 had significant general combining ability effects for days to flowering, days to maturity, internodal length, leaf area, and achene yield per plant. Among testers, A-26 and HBRS-1 were good general combiners for days to flowering, days to maturity, plant height, leaf area, head diameter, stem girth, percentage of filled achenes, 100-achene weight, and achene yield per plant. Crosses A-165 × A-26, A-41 $\times$ A-35, A-1 $\times$ G-12, and A-41 $\times$ HBPS-1 had significant and positive SCA effects for percentage of filled achenes, 100 achene weight, and achene yield per plant. Four best SCA crosses are recommended to be the best hybrids for cultivation. Non-additive type of gene action was found for all of the plant traits, which is desirable for heterosis breeding and may be exploited in hybrid seed production.

Key words: GCA, SCA, line $\times$ tester and yield.

$\mathrm{E}$ dible oil is major constituent of our diet but Pakistan is chronically deficient in its production, and large quantity of the country's edible oil requirements are met through imports. Oilseed sector, due to ever increasing consumption of edible oil, has attained critical importance in the economy of Pakistan. Total availability of edible oil during 2009-2010 was 1749 million tons, whereas local production stood at 0.680 million tons which accounted for $24 \%$ of the total availability while the remaining 1246 million tons was made available through imports (Economic Survey of Pakistan, 2009-2010).

This gap in the consumption and production can be filled by introducing cultivars with high edible oil contents, lodging resistance, high achenes yield, drought tolerance, and early maturity. Domestic production of edible oil can be increased by increasing the area and per acre yield of conventional and non-conventional oilseed crops. The area under oilseed crops cannot be increased as land resources are limited therefore, the only way left is the improvement of genetic potential of existing oilseed crops and introduction of new crops.

Sunflower (Helianthus annuus L.) appears to be the only crop which can play a crucial role in supplementing

\footnotetext{
${ }^{1}$ University of Agriculture, Faculty of Agriculture, Department of Plant Breeding \& Genetics, Faisalabad, Pakistan.

"Corresponding author (ms.ahmedgenes@gmail.com).

Received: 27 December 2010.

Accepted: 13 December 2011.
}

our local oil production due to its high yield potential, high edible oil contents, drought resistance, salt tolerance and alteration in the present cropping pattern. Being a short duration crop (90-110 d), it can be grown effectively twice a year under irrigation as well as rain fed conditions.

Presence of genetic variability is assessed as a first step for the development of high yielding and better adapted varieties/hybrids. It is essential to identify superior parents for hybridization and crosses to develop the genetic variability for selection of superior genotypes (Gangappa et al., 1997a). The line $\times$ tester analysis is one of the efficient methods of evaluating large number of inbred lines as well as providing information on the relative importance of general combining ability and specific combining ability effects for interpreting the genetic basis of important plant traits. The general combining ability (GCA) of a line means the average value of its performance in hybrids when crossed with other lines. The performance of individual hybrids is used to obtain specific combining ability (SCA), and that of the lines crossed to form that hybrid (Fick and Miller, 1997). Based on the combining ability analysis of different characters, higher SCA values refer to dominant gene effects and higher GCA effects indicate a greater role of additive gene effects controlling these characters. If both the GCA and SCA values are not significant, epistatic gene effects share an important role in determining these characters (Fehr, 1993).

In the present research study, parents and hybrids 
produced from line $\times$ tester mating were evaluated. The objective of this study was to estimate GCA and SCA of parents so as to identify superior combiners for high achene yield, early maturity, and lodging resistance.

\section{MATERIALS AND METHODS}

Fifteen genotypes/lines of sunflower were obtained from Oilseed Research Group, Department of Plant Breeding and Genetics, University of Agriculture Faisalabad (UAF), Pakistan. These were grown in field during spring 2009. Ten successively selfed accessions were used as female parents (lines) and five as male parents (testers) and crosses were attempted following line $\times$ tester fashion through controlled pollinations. In this way 50 crosses were developed for further study. The seeds of 50 crosses and their 15 parents were planted in field during September 2009, following a randomized complete block design with three replicates. The seeds were sown in $10 \mathrm{~m}$ long rows keeping $30 \mathrm{~cm}$ plant to plant and $75 \mathrm{~cm}$ row to row distance. All agronomic and cultural practices were performed uniformly from sowing till harvest. Ten plants of each entry in each replicate were taken at random and data were recorded for days to flowering, days to maturity, plant height, internodal length, and leaf area, number of leaves per plant, head diameter, stem girth, percentage of filled achenes, 100 achene weights, and achene yield per plant. The data were subjected to ANOVA according to Steel et al. (1997). Data were further analyzed for general and specific combining abilities, following Line $\times$ Tester analysis given by Kempthorne (1957). The significance of GCA and SCA effects was determined at the 0.05 and 0.01 levels using the t-test.

\section{RESULTS AND DISCUSSION}

Mean squares of all traits revealed significant differences among sunflower genotypes except 100 achene weight (Table 1). The differences among crosses were highly significant for all traits except internodal length, stem girth and 100 achene weight. Parents also differed significantly for all traits except 100 achene weight and internodal length. Highly significant differences existed for all traits except stem girth among lines and except internodal length among testers. However, line $\times$ tester interaction was significant for all characters except 100 achene weight and stem girth. Crosses vs. parents were significant for all characters under study. Significant difference within various components indicated the presence of genetic variability in the breeding material used in the study. This genetic variability may be exploited in the breeding programs for improvement of sunflower achene yield and its related traits. Significant differences among parents vs. crosses indicated the presence of heterosis in crosses that may be manifested for the development of high yielding sunflower hybrids. Significant differences have also been reported by early researchers among sunflower genotypes (Muppidathi et al., 1996; Monotti et al., 2000; Nehru et al., 2000; Gvozdenovic et al., 2005; Habib et al., 2007), males and females parents (Alone et al., 1996; Muppidathi et al., 1996; Gangappa et al., 1997b; Gill et al., 1998; Wang et al., 1997; Adefris et al., 1999; Jayalakshmi et al., 2000; Kannababu and Karivaratharaju, 2000; Monotti et al., 2000; Sharma et al., 2003; Habib et al., 2007), L × T interaction (Laureti and Del Gatto, 2001; Ortis et al., 2005; Binodh et al., 2008), and parents vs. crosses (Alone et al., 1996; Shekar et al., 1998; Ashoke et al., 2000; Habib et al., 2007; Khan et al., 2008) for achene yield and its components. Non-significant mean squared values for $\mathrm{L} \times \mathrm{T}$ interaction in days to maturity, plant height, head diameter, and seed yield have also been reported by Habib et al. (2007).

The mean sum of squares due to crosses was split into lines, testers and interactions. The lines were revealed to be significant for all the characters except stem girth, while the testers showed significant mean sum of squares for all the characters under studied apart from internodal length. The interaction mean sum of square was found to be significant for days to flowering, days to maturity, plant height, leaf area, number of leaves per plant, head diameter, percent filled achenes, and

Table 1. Mean squares from ANOVAs of indicated plant traits of 195 sunflower genotypes.

\begin{tabular}{|c|c|c|c|c|c|c|c|c|c|c|c|c|}
\hline SOV & DF & DFT & DTM & PH & IL & LA & NLP & HD & SG & $\% \mathrm{FA}$ & 100-WT & AYP \\
\hline Replicates & 2 & $4.5613^{* * *}$ & 0.32 & 2.39 & 0.71 & $67.13^{* *}$ & 1.85 & 3.80 & 0.33 & 1.23 & 0.19 & $7.425^{* *}$ \\
\hline Genotypes & 64 & $170.49^{* *}$ & $82.07^{* *}$ & $3282.40^{* * *}$ & $3.61^{* * *}$ & $14672.75^{* *}$ & $68.19^{* *}$ & $32.18^{* *}$ & $9.77^{* * *}$ & $124.59^{* * *}$ & 3.056 & $677.50^{* *}$ \\
\hline Crosses & 49 & $145.38^{* *}$ & $58.53^{* *}$ & $395.81^{* * *}$ & 2.91 & $4132.15^{* *}$ & $58.54^{* *}$ & $23.35^{* *}$ & 2.14 & $27.42^{* *}$ & 1.80 & $282.11^{* *}$ \\
\hline Parents & 14 & $172.60^{* *}$ & $109.06^{* * *}$ & $4529.28^{* * *}$ & 3.54 & $15130.01^{* *}$ & $47.06^{* *}$ & $32.48^{* *}$ & $4.98^{*}$ & $91.06^{* * *}$ & 1.08 & $90.30^{* * *}$ \\
\hline Lines (L) & 9 & $186.06^{* *}$ & $53.08^{* *}$ & $865.51^{* *}$ & $7.00^{*}$ & $9051.87^{* *}$ & $161.94^{* *}$ & $66.82^{* *}$ & 3.97 & $57.14^{* *}$ & $4.005^{*}$ & $592.33^{* *}$ \\
\hline Testers (T) & 4 & $348.92^{* *}$ & $79.81^{* *}$ & $741.14^{* *}$ & 3.30 & $18542.5^{* *}$ & $175.05^{* *}$ & $20.94^{* *}$ & $7.60^{*}$ & $107.29^{* * *}$ & $6.23^{*}$ & $607.71^{* *}$ \\
\hline $\mathrm{L} \times \mathrm{T}$ & 36 & $112.60^{* * *}$ & $57.52^{* *}$ & $242.26^{* *}$ & 1.84 & $1301.08^{* *}$ & $19.75^{* *}$ & $12.75^{* *}$ & 1.07 & $11.12^{* *}$ & 0.76 & $168.38^{* * *}$ \\
\hline Crosses vs. Parents & 1 & $1370.97^{* *}$ & $857.70^{* *}$ & $127269.37^{* *}$ & $38.65^{* * *}$ & $524760.00^{* *}$ & $836.41^{* *}$ & $460.97^{* *}$ & $450.75^{* *}$ & $5354.94^{* * *}$ & $91.84^{* * *}$ & $28272.52^{* *}$ \\
\hline Error & 128 & 3.45 & 5.70 & 3.30 & 0.19 & 52.82 & 1.71 & 0.52 & 0.3097 & 1.86 & 0.05 & 3.40 \\
\hline$\sigma^{2}$ gca & & 0.48 & 0.11 & 2.27 & 0.01 & 41.84 & 0.57 & 0.15 & 0.01 & 0.24 & 0.02 & 1.68 \\
\hline$\sigma^{2} \mathrm{sca}$ & & 36.19 & 16.96 & 80.03 & 0.55 & 410.98 & 5.99 & 4.07 & 0.24 & 3.22 & 0.24 & 55.03 \\
\hline$\sigma^{2} \mathrm{sca} / \sigma^{2} \mathrm{gca}$ & & 75.39 & 154.18 & 32.25 & 5.5 & 9.82 & 10.50 & 27.13 & 24 & 13.41 & 12 & 32.76 \\
\hline$\left[\sigma^{2} \mathrm{sca} / \sigma^{2}\right.$ gca $]$ & $1 / 2$ & 8.68 & 41.18 & 5.68 & 2.35 & 3.13 & 3.24 & 5.21 & 4.90 & 3.66 & 3.46 & 5.72 \\
\hline
\end{tabular}

*Significant at 0.05 level. ${ }^{* *}$ Significant at 0.01 level.

SOV: Source of variance; DF: Degree of freedom; DTF: Days to flowering; DTM: Days to maturity; PH; Plant height; IL: Internodal length; LA: Leaf area; NLP: Number of leaves per plant; HD: Head diameter; SG: stem girth; \%FA: \%filled achenes; 100-AW: 100-Achene weight; AYP: Achene yield per plant. 
achenes yield per plant. The variance component due to specific combining ability (SCA) was higher in amount than that of general combining ability (Table 1) for all the characters demonstrating prevalence of non-additive (dominant, overdominance and epistasis) type of gene action, and therefore, heterosis breeding may be rewarded which is in agreement with the findings of Shankar et al. (2007). Dominant gene effects were reported for days to flowering, number of leaves per plant, head diameter by Naik et al. (1999), Skoric et al. (2000) and Marinkovic et al. (2000). Over dominance effects for plant height, leaf area and 100-achene weight were also reported by Naik et al. (1999) and Skoric et al. (2000).

The general combining ability effects (Table 2) indicated that all the lines except A-179, G-5, and G-46 were good combiners for days to flowering and days to maturity. Maximum negatively significant GCA showed that these lines can be used for short duration hybrid progeny. These results were in accordance with the findings of Shankar et al. (2007). Apart from lines, A-41, A-165 and A-40, all the lines used under study depicted maximum negatively significant GCA effects for plant height and internodal length while highly significant value for leaf area and number of leaves per plant. While careful selection criteria should be used for stem girth because did not show maximum significance for stem girth. Short stature, less internodal length and large leaf area and maximum numbers of leaves per plant is prerequisite for high yielding lodging resistant hybrid progeny. All the lines revealed exceedingly momentous GCA effects for percent filled grain and achenes yield per plant except A-165 and A-179 while all the lines showed non-significant GCA results for 100-achene weight excluding G-5. These results are in contradiction with the finding of Goksoy et al. (2000). So, careful selection criteria should be adopted for 100-achene yield. All the lines revealed significant results for head diameter. However, these results do not match with the findings of Kaya and Atakisi (2004). Testers (Table 2) demonstrated highly significant GCA effects for all the traits under study with the exception of internodal length and 100-achene weight. These results are in compliance with the findings of Shankar et al. (2007). Testers with non-significant traits and low yield performance meet the general criteria for testing the general combining ability performance lines. The parents, which were good general combiners for economic qualities, might be extensively used in hybridization programs.

With the point of view of objectives of the present study selection criteria for identification of suitable hybrids with the parameters like; days to flowering, and days to maturity for early maturity, plant height, internodal length, leaf area and number of leaves per plant for lodging resistance and heat diameter, percent filled achenes, 100-achene weight and achene yield per plant for economic yield must be under consideration. Cross combinations (Table 3); A-165 × A-26 and A-41 $\times$ A-35 depicted excellent SCA performance for more than $80 \%$ characters under study, while A-1 $\times$ G- 12 and A-41 $\times$ HBPS-1 revealed highly significant SCA effects for more than $70 \%$ and 60 traits respectively. In the bulk of the crosses high SCA effects were due to low $\times$ low, high $\times$ low and low $\times$ high combining lines $\times$ testers which further demonstrate the operation of non-additive gene action for the traits under studied.

Hybrids (Table 3); G-5 × A-26, G-5 × A-35, A-27 × A-35, A- $29 \times$ A- 37, A- $65 \times$ A-26, A- $40 \times$ G- 12 and A- $40 \times$ A-26 illustrated high negatively significant SCA effects for days to flowering and days to maturity. These characters might be due to low $\times$ low, high $\times$ low and low $\times$ high combining parents indicating non-additive gene action so, heterosis breeding may be rewarded. These results were in conformity with the earlier findings of Bajaj et al. (1997), Lande et al. (1997), Kumar et al. (1998), Shekar et al.

Table 2. General combining ability effects of sunflower lines and testers for yield and its components.

\begin{tabular}{|c|c|c|c|c|c|c|c|c|c|c|c|}
\hline & DFT & DTM & PH & IL & LA & NLP & HD & SG & $\% \mathrm{FA}$ & 100-AW & AYP \\
\hline \multicolumn{12}{|l|}{ Lines } \\
\hline G-5 & -0.29 & 0.07 & $0.76^{*}$ & $0.72^{*}$ & $-38.26^{* *}$ & -0.56 & $-1.38^{* *}$ & $-0.46^{*}$ & $1.27^{* * *}$ & $1.10^{* * *}$ & $-9.40^{\text {*** }}$ \\
\hline A-179 & $2.77^{* *}$ & -0.07 & $-3.12^{* *}$ & $-0.29^{*}$ & $-23.21^{* *}$ & $3.17^{*}$ & $-3.50^{* *}$ & $0.57^{*}$ & $-2.46^{* * *}$ & 0.21 & 0.09 \\
\hline A-1 & $-2.36^{* *}$ & $-1.53^{* *}$ & $-4.25^{* *}$ & $0.73^{*}$ & $1.50^{* *}$ & $-6.76^{* *}$ & $1.54^{* *}$ & -0.04 & $-2.33^{* *}$ & -0.18 & $-7.29^{* *}$ \\
\hline A-27 & $-5.36^{* *}$ & $-1.80^{* *}$ & $-2.01^{* *}$ & $-0.58^{*}$ & $17.61^{* *}$ & $-4.16^{*}$ & $2.94^{* *}$ & 0.05 & $-0.93^{*}$ & -0.11 & $-1.14^{* * *}$ \\
\hline A-7 & $4.17^{* *}$ & $3.40^{* *}$ & $-2.69^{* * *}$ & $0.55^{*}$ & $27.43^{* *}$ & 0.77 & $-1.51^{* *}$ & -0.02 & $-2.06^{* * *}$ & $-0.65^{*}$ & $2.51^{* * *}$ \\
\hline A-41 & $-1.29^{* *}$ & $-1.40^{* *}$ & $-5.05^{* *}$ & 0.01 & $4.61^{* *}$ & -0.43 & 0.56 & $0.89^{*}$ & $0.61^{*}$ & -0.29 & $5.58^{* * *}$ \\
\hline A-39 & $-0.96^{* *}$ & $2.00^{* *}$ & $-4.23^{* * *}$ & $-1.46^{*}$ & $-11.27^{* * *}$ & 0.17 & $2.53^{* *}$ & $-0.96^{*}$ & $0.27^{*}$ & -0.03 & $-2.52^{* * *}$ \\
\hline A-165 & $-2.83^{* *}$ & $-2.53^{* *}$ & 0.53 & -0.18 & $-24.71^{* *}$ & $3.24^{*}$ & $-1.62^{* *}$ & $-0.29^{*}$ & 0.07 & $0.61^{*}$ & $3.71^{* *}$ \\
\hline A-40 & $6.44^{* *}$ & 0.40 & $20.74^{* * *}$ & -0.02 & $6.81^{* *}$ & $3.31^{*}$ & $-1.04^{* *}$ & 0.20 & $2.41^{\text {*** }}$ & -0.40 & $-3.50^{* * *}$ \\
\hline G-46 & -0.29 & $1.47^{* *}$ & $-0.68^{*}$ & $0.50^{*}$ & $39.48^{* *}$ & 1.24 & $1.49^{* *}$ & 0.06 & $3.14^{* * *}$ & -0.25 & $11.95^{\text {** }}$ \\
\hline Standard error & 0.51 & 0.66 & 0.37 & 0.18 & 0.11 & 2.13 & 0.34 & 0.05 & 0.16 & 0.31 & 0.47 \\
\hline \multicolumn{12}{|l|}{ Testers } \\
\hline G-12 & $-2.09^{* *}$ & $-1.77^{* *}$ & $-2.60^{* * *}$ & $0.23^{*}$ & $-9.69^{* *}$ & $-2.43^{* *}$ & $0.65^{*}$ & $-0.33^{*}$ & $-1.29^{* *}$ & -0.27 & $-4.40^{\text {** }}$ \\
\hline A-26 & $-3.63^{* *}$ & $-1.33^{* *}$ & $-0.72^{*}$ & $-0.47^{*}$ & $0.11^{*}$ & $-2.03^{* *}$ & $0.46^{*}$ & $-0.37^{*}$ & $-0.86^{*}$ & $-0.55^{*}$ & $3.13^{* *}$ \\
\hline A-35 & $-1.29^{* *}$ & -0.27 & $3.21^{* *}$ & $0.35^{*}$ & $-8.03^{* *}$ & $1.57^{*}$ & $-0.49^{*}$ & $-0.19^{*}$ & $-0.96^{*}$ & 0.09 & $-3.26^{* * *}$ \\
\hline A-57 & $2.34^{* *}$ & $1.73^{* *}$ & $-6.32^{* *}$ & 0.06 & $-24.05^{* *}$ & $3.27^{* *}$ & $-1.24^{* *}$ & 0.04 & -0.19 & 0.06 & $-1.67^{* * *}$ \\
\hline HBRS-1 & $4.67^{* *}$ & $1.63^{* *}$ & $6.43^{* *}$ & -0.18 & $41.66^{* *}$ & -0.39 & $0.62 *$ & $0.85^{*}$ & $3.31^{* * *}$ & $0.67^{* * *}$ & $6.20^{\text {*** }}$ \\
\hline Standard error & 0.37 & 0.47 & 0.27 & 0.13 & 0.08 & 1.51 & 0.24 & 0.04 & 0.11 & 0.21 & 0.33 \\
\hline
\end{tabular}

"Significant at 0.05 level. ${ }^{* *}$ Significant at 0.01 level.

DTF: Days to flowering; DTM: Days to maturity; PH: Plant height; IL: Internodal length; LA: leaf area; NLP: number of leaves per plant: HD: head diameter; SG: stem girth; \%FA: \% filled achenes; 100-AW: 100-Achene weight; AYP: Achene yield per plant. 
Table 3. Specific combining ability effects of $\mathbf{5 0}$ sunflower crosses for yield and related traits.

\begin{tabular}{|c|c|c|c|c|c|c|c|c|c|c|c|}
\hline Crosses & DFT & DTM & $\mathrm{PH}$ & IL & LA & NLP & HD & SG & $\% \mathrm{FA}$ & 100-AW & AYP \\
\hline $\mathrm{G}-5 \times \mathrm{G}-12$ & -3.37 & 1.70 & $17.15^{* * *}$ & $0.53^{*}$ & $-12.48^{*}$ & $0.83^{* *}$ & 0.40 & 0.62 & 0.49 & $-0.18^{*}$ & -3.25 \\
\hline $\mathrm{G}-5 \times \mathrm{A}-26$ & $-7.51^{*}$ & $-4.73^{*}$ & -0.96 & -0.19 & $17.89^{*}$ & $-1.57^{* *}$ & 0.26 & 0.12 & 2.73 & $-0.68^{*}$ & 2.24 \\
\hline $\mathrm{G}-5 \times \mathrm{A}-35$ & $6.49^{*}$ & $2.87^{*}$ & 0.74 & $-0.52^{*}$ & -14.75 & $-3.84^{* *}$ & $-2.39^{*}$ & -0.59 & $-2.51^{*}$ & $0.48^{*}$ & -0.02 \\
\hline $\mathrm{G}-5 \times \mathrm{A}-57$ & 2.19 & -0.47 & 1.70 & $-0.53^{*}$ & 10.21 & $2.13^{* *}$ & 0.23 & -0.51 & -0.94 & $0.45^{*}$ & 4.05 \\
\hline G-5 $\times$ HBRS -1 & 2.19 & 0.63 & $-18.85^{* * *}$ & $0.74^{*}$ & 9.09 & $2.46^{* *}$ & 1.50 & -0.80 & 0.23 & -0.06 & -3.02 \\
\hline A-179 × G-12 & $8.23^{* *}$ & -1.83 & -3.37 & 0.31 & -1.00 & $-0.57^{* *}$ & $-2.03^{*}$ & 0.59 & 0.23 & 0.02 & 1.69 \\
\hline A-179 $\times$ A-26 & -3.91 & $-2.60^{*}$ & $13.78^{* * *}$ & $-1.2 * *$ & 2.79 & $1.69^{* * *}$ & 1.04 & -0.85 & 0.79 & $-0.41^{*}$ & $6.33^{*}$ \\
\hline A-179 $\times$ A-35 & -2.57 & $5.00^{* *}$ & -5.55 & $0.69^{*}$ & 9.60 & $1.09^{* *}$ & 1.13 & -0.81 & -1.11 & $-0.24^{*}$ & $7.48^{*}$ \\
\hline A-179 $\times$ A-57 & -0.21 & -0.00 & -2.96 & -0.25 & -0.24 & $-1.94^{* *}$ & -1.49 & -0.85 & 0.13 & $-0.22^{*}$ & $-9.94^{* *}$ \\
\hline A- $179 \times$ HBRS- 1 & -1.54 & -0.57 & -1.90 & $0.59^{*}$ & -11.15 & $-1.81^{* *}$ & 1.35 & 0.90 & -0.04 & $0.86^{*}$ & $-5.55^{*}$ \\
\hline $\mathrm{A}-1 \times \mathrm{G}-12$ & $-5.31^{* *}$ & $-4.70^{* *}$ & $-11.68^{* *}$ & -0.27 & $-28.38^{* * *}$ & $1.69^{* *}$ & 0.75 & 0.13 & $-2.24^{*}$ & $0.36^{*}$ & $8.14^{*}$ \\
\hline A-1 $\times$ A-26 & 2.89 & $6.87^{* *}$ & -1.43 & 0.23 & $-19.91^{*}$ & $-0.37^{* *}$ & 0.97 & 1.01 & $-2.34^{*}$ & $0.33^{*}$ & -7.52 \\
\hline $\mathrm{A}-1 \times \mathrm{A}-35$ & -0.11 & $-4.87^{* *}$ & -0.82 & $0.64^{*}$ & $57.90^{* * *}$ & $3.69^{* *}$ & 0.56 & 1.06 & $3.43^{* * *}$ & $-0.91^{*}$ & -2.13 \\
\hline A-1 $\times$ A-57 & 5.93 & 1.80 & $9.04^{*}$ & 0.10 & $22.22^{* * *}$ & $-3.34^{* *}$ & 0.69 & -0.34 & 1.66 & 0.02 & $9.67 * *$ \\
\hline A- $1 \times$ HBRS -1 & 3.41 & 0.90 & 4.89 & -0.13 & $12.60^{*}$ & $-1.67^{* *}$ & -1.59 & -0.62 & -1.51 & $0.21^{*}$ & $-8.16^{*}$ \\
\hline $\mathrm{A}-27 \times \mathrm{G}-12$ & 4.03 & -2.10 & $8.22^{*}$ & -0.03 & -5.53 & $-1.24^{* *}$ & -0.32 & -0.71 & -1.64 & $0.39^{*}$ & $4.66^{*}$ \\
\hline A- $27 \times A-26$ & 3.56 & $4.47^{* *}$ & -5.80 & $0.81^{*}$ & $-11.94^{*}$ & $0.69^{* *}$ & $-2.59^{*}$ & 0.11 & $-2.41^{*}$ & $0.23^{*}$ & 4.26 \\
\hline A- $27 \times$ A-35 & $-9.11^{* *}$ & $-4.60^{* * *}$ & -6.16 & 0.08 & $-15.51^{* * *}$ & $1.22^{* *}$ & -0.01 & -0.70 & 1.03 & 0.08 & $-13.90^{* * *}$ \\
\hline A- $27 \times$ A-57 & 0.59 & $2.73^{*}$ & -1.14 & $-0.66^{*}$ & 7.27 & $0.39^{* *}$ & 1.74 & 1.07 & 0.93 & 0.03 & -1.48 \\
\hline A-27 $\times$ HBRS -1 & 0.93 & -0.50 & 4.88 & $0.58^{*}$ & $25.71^{* * *}$ & $2.83^{* *}$ & 1.18 & -1.11 & 2.09 & $-0.73^{*}$ & $6.45^{*}$ \\
\hline $\mathrm{A}-7 \times \mathrm{G}-12$ & 5.16 & 0.03 & -4.43 & $0.61^{*}$ & -6.91 & $4.83^{* *}$ & -0.73 & 0.01 & 0.49 & $-1.06^{*}$ & $14.71^{* *}$ \\
\hline A-7 $\times$ A-26 & 2.36 & $-2.73^{*}$ & 2.78 & $0.71^{*}$ & $14.61^{*}$ & $2.76^{* * *}$ & 0.17 & 0.65 & 0.73 & $0.14^{*}$ & $-14.33^{* *}$ \\
\hline A-7 $\times$ A-35 & -3.31 & 0.53 & 2.39 & 0.15 & 0.43 & $-1.84^{* *}$ & 0.11 & 0.93 & 0.16 & $0.55^{*}$ & 2.90 \\
\hline A-7 $\times A-57$ & -0.94 & $2.53^{*}$ & 0.05 & $-0.56^{*}$ & $-30.61^{* *}$ & $-3.54^{* *}$ & 1.59 & -1.32 & 1.39 & $0.35^{*}$ & $-4.96^{*}$ \\
\hline A-41 $\times$ A-26 & -3.27 & -0.37 & 4.77 & $-0.79^{*}$ & $22.48^{* * *}$ & $-2.21^{* *}$ & -1.14 & 0.96 & -2.77 & 0.02 & 1.69 \\
\hline A-41 $\times$ A-35 & $7.63^{*}$ & $4.17^{* *}$ & $13.69^{* * *}$ & $-0.52^{*}$ & $23.34^{* *}$ & $-0.31^{* *}$ & $2.79^{* * *}$ & -0.63 & 1.16 & $-0.47^{*}$ & $-6.94^{*}$ \\
\hline A-41 $\times$ A-57 & -1.17 & $-5.27^{* *}$ & $-8.23^{*}$ & -0.37 & 9.61 & $0.29^{* *}$ & -1.59 & -0.24 & -1.61 & $-0.31^{*}$ & 3.23 \\
\hline A-41 $\times$ HBRS- 1 & $6.16^{* *}$ & $3.00^{*}$ & 1.01 & 1.33 & $-13.38^{*}$ & $-1.97^{* *}$ & 2.33 & 0.69 & $1.83^{*}$ & $0.25^{*}$ & $4.98^{*}$ \\
\hline A- $39 \times \mathrm{G}-12$ & $-13.47^{* *}$ & 1.33 & $-21.93^{* * *}$ & 0.16 & $23.65^{* *}$ & $4.66^{* *}$ & -0.65 & 0.80 & 0.39 & $0.72^{*}$ & $6.74^{*}$ \\
\hline A-39 $\times$ A- 26 & 0.86 & -3.23 & $6.46^{*}$ & $-0.60^{*}$ & $43.24^{* *}$ & $-2.67^{* * *}$ & $-2.85^{*}$ & 0.89 & -1.77 & -0.04 & $-8.02^{*}$ \\
\hline $\mathrm{A}-39 \times \mathrm{A}-35$ & -3.71 & -0.57 & -5.46 & $0.65^{*}$ & $24.66^{* *}$ & $0.09^{*}$ & -1.61 & -0.08 & -0.84 & $-0.19^{*}$ & $-7.40^{*}$ \\
\hline A-39 $\times$ A -57 & $6.49^{* *}$ & $6.00^{* *}$ & 4.42 & $1.23^{* * *}$ & -8.97 & $-1.97^{* *}$ & -0.72 & -1.19 & 1.39 & $0.27^{*}$ & -4.16 \\
\hline A-39 $\times$ HBRS- 1 & 4.49 & $-2.07^{*}$ & $9.03^{*}$ & $-1.1^{* *}$ & -9.15 & $2.76^{* * *}$ & -0.37 & 0.91 & 0.49 & $0.69^{*}$ & 3.40 \\
\hline A-165 × G-12 & 4.53 & -0.07 & -0.94 & 0.09 & $44.02^{* *}$ & $-0.61^{* *}$ & 1.31 & 0.93 & $-2.94^{*}$ & $-0.23^{*}$ & -2.86 \\
\hline A-165 × A-26 & $-11.81^{* *}$ & $-3.30^{*}$ & -7.06 & $-0.67^{*}$ & $22.52^{* *}$ & $-0.27^{* *}$ & 1.39 & -0.20 & $1.89^{*}$ & $-0.64^{*}$ & $11.01^{* * *}$ \\
\hline A-165 × A-35 & -3.51 & $-3.37^{*}$ & $-13.85^{* *}$ & -0.30 & $26.39^{* *}$ & $-2.64^{* *}$ & 1.12 & -0.17 & -1.31 & $-0.08^{*}$ & $-4.76^{*}$ \\
\hline A-165 × A-57 & 2.36 & $-4.47^{* * *}$ & $10.00^{* * *}$ & $0.64^{*}$ & -5.97 & $-0.71^{* *}$ & -0.07 & -0.73 & 1.59 & $1.05 * *$ & -2.06 \\
\hline A-165× HBRS-1 & -5.31 & 1.80 & -3.63 & -0.35 & 6.17 & $0.36^{* *}$ & $4.35^{* *}$ & -0.89 & -0.31 & -0.03 & $5.65^{*}$ \\
\hline A-40 $\times$ G-12 & -5.27 & $-7.20^{* *}$ & 4.93 & 0.17 & 30.91 & $-1.68^{* * *}$ & $-2.43^{*}$ & 1.21 & -1.07 & $-0.17^{*}$ & -0.16 \\
\hline $\mathrm{A}-40 \times \mathrm{A}-26$ & $11.73^{* *}$ & $13.23^{* *}$ & 2.55 & $0.89^{* *}$ & $-24.77^{* *}$ & $3.66^{* *}$ & -1.73 & -0.86 & 1.09 & $-0.58^{*}$ & 1.34 \\
\hline $\mathrm{A}-40 \times \mathrm{A}-35$ & -0.44 & 2.03 & -2.23 & $-0.91^{*}$ & -0.85 & $-1.37^{* *}$ & 1.20 & -1.31 & -1.64 & $-0.26^{*}$ & -2.76 \\
\hline $\mathrm{A}-40 \times \mathrm{A}-57$ & -0.57 & $2.27^{*}$ & -4.52 & $9.96^{* *}$ & $-22.2^{* *}$ & $2.93^{* *}$ & 0.12 & $1.58^{*}$ & -0.07 & $-0.33^{*}$ & -0.35 \\
\hline A-40 $\times$ HBRS- 1 & -0.24 & -0.13 & -1.47 & 0.12 & $55.44^{* *}$ & $2.59^{* *}$ & -2.00 & -0.53 & 1.60 & 0.10 & $-4.52^{*}$ \\
\hline $\mathrm{G}-46 \times \mathrm{G}-12$ & 2.13 & $-2.80^{*}$ & 5.05 & 0.35 & -2.38 & $-1.31^{* *}$ & $2.62^{*}$ & 0.03 & $2.26^{*}$ & -0.09 & 3.45 \\
\hline $\mathrm{G}-46 \times \mathrm{A}-26$ & -0.87 & -1.37 & 3.17 & $-1.34^{* * *}$ & $10.87^{*}$ & $3.29^{* * *}$ & -1.94 & 0.99 & 0.76 & $-0.34^{*}$ & 4.18 \\
\hline $\mathrm{G}-46 \times \mathrm{A}-35$ & $-8.71^{* *}$ & $4.63^{* *}$ & 1.96 & $0.83^{*}$ & $13.50^{*}$ & $-0.31^{* *}$ & -0.30 & -0.78 & 4.29 & $0.34^{*}$ & -4.10 \\
\hline $\mathrm{G}-46 \times \mathrm{A}-57$ & -4.51 & 0.20 & -4.70 & $-2.17^{* * *}$ & 4.08 & $3.29^{* *}$ & $2.39^{*}$ & -0.53 & -0.81 & -0.05 & $12.37^{* *}$ \\
\hline G-46 $\times$ HBRS- 1 & 3.49 & -1.53 & -4.55 & $-0.69^{*}$ & $-15.87^{*}$ & $-0.31^{* *}$ & $-3.70^{*}$ & -0.14 & -1.71 & $0.29^{*}$ & -3.85 \\
\hline Standard error & 4.53 & 2.13 & 6.20 & 0.49 & -11.69 & -0.01 & -2.21 & 1.16 & -1.81 & -0.10 & -4.50 \\
\hline
\end{tabular}

*Significant at 0.05 level. ${ }^{* *}$ Significant at 0.01 level.

DTF: Days to flowering; DTM: Days to maturity; PH: Plant height; IL: Internodal length; LA: leaf area; NLP: number of leaves per plant; HD: head diameter; SG: stem girth; \%FA: \%filled achenes; 100-AW: 100-Achene weight; AYP: Achene yield per plant.

(1998), Goksoy et al. (1999), Gomez et al. (1999), Naik et al. (1999), Nirmala et al. (1999), Radhika et al. (1999), Goksoy and Turan (2005), Gvozdenovic et al. (2005), Ortis et al. (2005), Hladni et al. (2006).

Hybrid combinations (Table 3); G-5 × G-12, A-27 $\times$ HBRS-1, A-41 × A-35, A-39 × A-26, and A-165 × A-26 gained maximum significance SCA effects for all the lodging resistant traits under studied viz; plant height, internodal length, leaf area, number of leaves per plant. None of the hybrid showed considerable performance for stem girth except A-39 × A-57, A-40 × A-35, and A-40 × A-57. So, it can be concluded that all the lines and testers used in this study are not suitable for producing hybrids with robust stem girth apart from progenitors of crosses; A-39 $\times$ A-57, A-40 $\times$ A-35, and A-40 $\times$ A-57.
Hybrid progeny; A-179 $\times$ HBRS-1, A-41 $\times$ HBRS- 1 and A-165 × A-26 demonstrated highly significant SCA effects for all three economic yield related traits viz; $\%$ filled achenes, 100-Achene weight, achene yield per plant. These results are in accordance with the findings of Lande et al. (1997); Lokendra et al. (1997); Kumar et al. (1998); Goksoy et al. (1999); Ashoke et al. (2000); Sharma et al. (2003); Gvozdenovic et al. (2005); Ortis et al. (2005); Hladni et al. (2006). They reported additive gene effects for plant height and leaf area.

\section{CONCLUSION}

According to above research study, it is concluded that breeding material evaluated has adequate genetic 
variability that may be exploited in further breeding programs. SCA and GCA ANOVAs proposed these characters under control of non-additive gene action. Further analysis revealed over-dominant gene action controlling these plant traits. Therefore, heterosis breeding is suggested for improvement in yield and related traits using this sunflower breeding material.

Among the lines, A-1, A-27, and A-39 showed maximum GCA effects and considered to be good general combiner for almost $80 \%$ traits under study these lines can be used for further heterosis breeding programs. A-26 and HBRS-1 found to be good general combiner testers. Cross combinations; A-165 $\times$ A-26, A-41 $\times$ A-35, A-1 $\times$ G-12, and A-41 $\times$ HBPS- 1 depicted excellent SCA performance for more than $80 \%$ traits under study.

Finally, it is concluded that non-additive type of gene action was found for all of the plant traits, which is desirable for heterosis breeding and may be exploited in hybrid seed production.

\section{Análisis de habilidad combinada para rendimiento de aquenios y características relacionadas en maravilla} (Helianthus annuus L.). Diez líneas de maravilla (Helianthus annuus L.), cinco verificadores, y 50 cruzas desarrolladas en la forma línea $\times$ verificador fueron evaluadas para efectos de capacidad de combinación general (GCA) y capacidad de combinación específica (SCA) en un diseño de bloques completos al azar en triplicado, en Faisalabad, durante 2009-2010. La variabilidad genética entre genotipos se evaluó con días a floración, días a madurez, altura de planta, longitud entre nudos, área foliar, número de hojas por planta, diámetro de cabeza, circunferencia del tallo, porcentaje de aquenios llenos, peso de 100 aquenios, producción de aquenios por planta. A-1, A-7, A-27, y A-39 tuvieron efectos significativos de GCA para días a floración, días a madurez, longitud entre nudos, área foliar, y producción de aquenios por planta. Entre los verificadores, A-26 y HBRS-1 fueron buenos combinadores generales para días a floración, días a madurez, altura de planta, área foliar, diámetro de cabeza, circunferencia del tallo, porcentaje de aquenios llenos, peso de 100 aquenios, y producción de aquenios por planta. Las cruzas A-165 × A-26, A-41 $\times$ A-35, A-1 × G-12, y A-41 × HBPS-1 tuvieron efectos SCA significativos y positivos para porcentaje de aquenios llenos, peso de 100 aquenios, y producción de aquenios por planta. Cuatro cruzas fueron recomendadas como los mejores híbridos para cultivo. No se encontró acción génica de tipo aditiva para ningún rasgo, lo cual es deseable para heterosis y puede ser explotado en producción de semillas híbridas.

Palabras clave: GCA, SCA, línea $\times$ verificador $y$ rendimiento.

\section{LITERATURE CITED}

Adefris, T., H. Jayaramaiah, S. Ramesh, and A. Teklewold. 1999. Genetic variability studies in sunflower (Helianthus annuиs L.) Crop Improvement 26:236-240.

Alone, R.K., R.D. Patil, S.N. Mate, and M.R. Manjare. 1996. Combining ability in sunflower. Indian Journal of Agricultural Research 30(3-4):215-220.

Ashoke. S., S.N. Mohamed, and S.L. Narayanan. 2000. Combining ability studies in sunflower (Helianthus annuus L.) Crop Research Hisar 20:457-462

Bajaj, R.K., K.K. Aujia, and G.S. Chahal. 1997. Combining ability studies in sunflower (Helianthus annuиs L.) Crop Improvement 24:50-54.

Binodh, A.K., N. Manivannan, and P.V. Varman. 2008. Combining ability analysis for yield and its contributing characters in sunflower (Helianthus annuus L.) Madras Agricultural Journal 95:295-300.

Economic Survey of Pakistan. 2009-2010. Agriculture. Chapter 10/2. Economic Survey of Pakistan. Finance Division, Economic Advisor's Wing, Government of Pakistan, Islamabad, Pakistan.

Fick, G., and J.F. Miller. 1997. Sunflower breeding. p. 397-439. In A.A. Schneiter (ed.) Sunflower technology and production. American Society of Agronomy (ASA), Madison, Wisconsin, USA

Gangappa, E., K.M. Channakrishnaiah, M.S. Harini, and S. Ramesh. 1997b. Studies on combining ability in sunflower (Helianthus annuus L.) Helia 20(27):73-84.

Gangappa, E., K.M. Channakrishnaiah., S. Rajesh, and M.S. Harini. 1997a. Exploitation of heterosis in sunflower (Helianthus annuus L.) Crop Research Hisar 13:339-348.

Gill, H.S., S.R. Khurana, T.P. Yadava, and R.K. Sheoran. 1998. Expression of heterosis for different characters in sunflower over environments. Haryana Agricultural University Journal of Research 28:95-100.

Goksoy, A.T., and Z.M. Turan. 2005. Combining abilities of certain characters and estimation of hybrid vigour in sunflower (Helianthus annuus L.) Acta Agronomica Hungarica 54:361-368.

Goksoy, A.T., A. Turkec, and Z.M. Turan. 1999. A study of some agronomical characteristics of synthetic varieties obtained from inbred lines of sunflower (Helianthus annuus L.) Turkish Journal of Agriculture and Forestry 23:349-354

Goksoy, A.T., A. Turkec, and Z.M. Turan. 2000. Heterosis and combining ability in sunflower (Helianthus anпиus L.) Indian Journal of Agricultural Science 70:525-529.

Gomez, S.D., M. Baldini, C.D. Aguilera, and G.P. Vannozzi. 1999. Genetic variances and heritability of sunflower traits associated with drought tolerance. Helia 22(31):23-34.

Gvozdenovic, S., J. Joksimovic, and D. Škoric. 2005. Gene effect and combining abilities for plant height and head diameter in sunflower. Genetika 37:57-64.

Habib, H., S.S. Mehdi, A. Rashid, M. Zafar, and M.S. Anjum. 2007. Heterosis and heterobeltosis studies for flowering traits, plant height and seed yield in sunflower. International Journal of Agriculture and Biology 9:355-358.

Hladni, N., D. Skoric, M. Kraljevic-Balalic, Z. Sakac, and D. Jovanovic. 2006. Combining ability for oil content and its correlations with other yield components in sunflower (Helianthus annuиs L.) Helia 29(44):101-110.

Jayalakshmi, V., B. Narendra, V. Sridhar, and K.R. Devi. 2000. Heterosis in sunflower (Helianthus annuus L.) Agricultural Science Digest 20:114-115.

Kannababu, N., and T.V. Karivaratharaju. 2000. Maternal influence of cytoplasmic genic male sterile lines on seed quality in sunflower (Helianthus annuus L.) Indian Journal of Plant Physiology 5:159162.

Kaya, Y., and I. K. Atakisi. 2004. Combining ability analysis of some yield characters of sunflower (Helianthus annuus L.) Helia 27(41):75-84

Kempthorne, O. 1957. Introduction to genetic statistics. John Wiley and Sons, New York, USA 
Khan, H., H. Rahman, H. Ahmad, H. Ali, Inamullah, and M. Alam. 2008. Magnitude of heterosis and heritability in sunflower over environments. Pakistan Journal of Botany 40:301-308.

Kumar, A.A., M. Ganesh, and P. Janila. 1998. Combining ability analysis for yield and yield contributing characters in sunflower (Helianthus annuus L.) Annals of Agricultural Research 19:437440.

Lande, S.S., D.G. Weginwar, M.C. Patel, A.R. Imbore, and P.W. Khorgade. 1997. Gene action, combining ability in relation to heterosis in sunflower (Helianthus annuus L.) through line $\times$ tester analysis. Journal of Soil and Crops 7:205-207.

Laureti, D., and A. Del Gatto. 2001. General and specific combining ability in sunflower (Helianthus annuus L.). Helia 24(34):1-16.

Lokendra, K., M. Singh, R.K. Sheoran, and L. Kumar. 1997. Inheritance of achene yield and its components in a sunflower population. Journal of Oilseeds Research 14:168-171.

Marinkovic, R., D. Skoric, B. Dozet, and D. Jovanovic. 2000. Effect of PET1 and ANN5 cytoplasms on some quantitative traits in sunflower lines and hybrids. Helia 23(32):73-86.

Monotti, M., M.A. Del Pino, D. Laureti, and S. Pieri. 2000. Trials of sunflower varieties in 1999. Info Agrario 56(11):35-43.

Muppidathi, N., R. Sankarapandian, and S. Rajarathinam. 1996. Stability and selection parameters in sunflower hybrids under rainfed vertisols. Madras Agricultural Journal 83(12):747-750.

Naik, V.R., S.R. Hiremath, and J.K. Girira. 1999. Gene action in sunflower. Karnataka Journal of Agricultural Sciences 12(1-4):4347.

Nehru, S.D., A. Manjunath, and D. Basavaraja. 2000. Extent of heterosis for seed yield and oil content in sunflower. Karnataka. Journal of Agricultural Science 13(3):718-720.
Nirmala, V., A. Gopalan, and D. Sassikumar. 1999. Correlation and path coefficient analysis in sunflower. Madras Agricultural Journal 86:269-272.

Ortis, L., G. Nestares, E. Frutos, and N. Machado. 2005. Combining ability analysis for agronomic traits in sunflower (Helianthus annuus L.) Helia (28):125-134.

Radhika, P., K. Jagadeshwar, and P.S. Sharma. 1999. Genetic analysis of seed yield and certain physiological parameters in sunflower. Journal of Research ANGRAU 27(1-2):5-17.

Shankar, V., G. Ganesh, M. Ranganatha, A.R.G. Suman, and A.V. Sridhar. 2007. Combining ability studies in diverse CMS sources in sunflower (Helianthus annuus L.) Indian Journal of Agricultural Research 41:171-176.

Sharma, S., R.K. Bajaj, N. Kaur, and S.K. Sehgal. 2003. Combining ability studies in sunflower (Helianthus annuus L.) Crop Improvement 30:69-73.

Shekar, G.C., H. Jayaramaiah, K. Virupakshappa, and B.N. Jagadeesh. 1998. Combining ability of high oleic acid in sunflower. Helia 21(28):7-14

Skoric, D., S. Jocic, and I. Molnar. 2000. General (GCA) and specific (SCA) combining abilities in sunflower. Zbornik naucnih radova 6:97-105

Steel, R.G.D., J.H. Torrie, and D.A. Dicky. 1997. Principles and procedures of statistics. A biometrical approach. ${ }^{2 n d}$ ed. p. $204-$ 227. McGraw Hill Book, Tuas Basin Link, Singapore.

Wang, P., J. Ji, Z.L. Chen, J.Y. Zang, Y. Wu, and G. Wang. 1997. Genetic analysis of main agronomic characters in oil sunflower (Helianthus annuus L.) Hereditas (Beijing) 19(6):15-17. 